\title{
Small bowel metastasis from pancreatic cancer in a long-term survival patient with synchronous advanced malignant pleural mesothelioma: A case report and literature review
}

\author{
MORENA FASANO $^{1 *}$, CARMINIA MARIA DELLA CORTE $^{1 *}$, GIOVANNI VICIDOMINI $^{2}$, \\ VALERIO SCOTTI ${ }^{3}$, PIER FRANCESCO RAMBALDI ${ }^{4}$, ALFONSO FIORELLI ${ }^{2}$, MARINA ACCARDO ${ }^{5}$, \\ FERDINANDO DE VITA $^{1}$, MARIO SANTINI ${ }^{2}$, FORTUNATO CIARDIELLO ${ }^{1}$ and FLORIANA MORGILLO ${ }^{1}$ \\ ${ }^{1}$ Division of Medical Oncology, Department of Clinical and Experimental Medicine 'F. Magrassi e A. Lanzara', \\ Second University of Naples; ${ }^{2}$ Thoracic Surgery Unit, Second University of Naples, I-80131 Naples; \\ ${ }^{3}$ Malzoni Radiosurgery Center, Neuromed Group, Agropoli, I-84100 Salerno; \\ Departments of ${ }^{4}$ Nuclear Medicine Division and ${ }^{5}$ Morphopathology, Second University of Naples, I-80131 Naples, Italy
}

Received March 11,2015; Accepted March 24, 2016

DOI: $10.3892 / 01.2016 .5279$

\begin{abstract}
Diffuse malignant pleural mesothelioma (MPM) is an aggressive tumor that originates from the surface of the pleura. Approximately $70 \%$ of cases are associated with chronic asbestos exposure. MPM is regarded as an incurable disease, with a median survival of $\sim 2$ years following intensive multimodality treatment. Pancreatic cancer is a malignancy also associated with a poor prognosis, with only $2 \%$ of patients surviving for 5 years. The majority of patients with pancreatic cancer are diagnosed with an advanced stage of disease and experience a poor response to therapy. The development of synchronous MPM and other types of cancer is rare. The present study describes a patient with synchronous, biphasic MPM and pancreatic adenocarcinoma, who was treated with a multimodal therapeutic approach with stereotactic body radiation therapy. Due to a suspected diagnosis of 'acute abdomen', an emergency small intestine resection was performed and a subsequent diagnosis of moderately-differentiated adenocarcinoma was confirmed. During a further immunohistochemical examination, pathologists determined that the small bowel metastasis descended from pancreatic cancer. The onset of bowel metastasis is an event rarely associated with MPM, and has not been previously described in the literature for cases of
\end{abstract}

Correspondence to: Dr Floriana Morgillo, Division of Medical Oncology, Department of Clinical and Experimental Medicine 'F. Magrassi e A. Lanzara', Second University of Naples, 5 Sergio Pansini Street, Building 16, I-80131 Naples, Italy

E-mail: florianamorgillo@yahoo.com

*Contributed equally

Key words: diffuse malignant pleural mesothelioma, pancreatic cancer, synchronous tumors, stereotactic body radiation therapy, multimodal chemoradiotherapic approach pancreatic cancer. Therefore, to the best of our knowledge, the present study describes the first case of intestinal metastasis from pancreatic cancer in a long-term survival patient with biphasic MPM.

\section{Introduction}

Diffuse malignant pleural mesothelioma (MPM) is an aggressive, rare tumor that originates from the surface of the pleura. Although relatively uncommon, the incidence of MPM is expected to increase in the next 20 years (1). Asbestos exposure is a major risk factor, with $70 \%$ of MPM patients presenting with a defined history of chronic asbestos exposure (1). MPM is regarded as an incurable disease, with a median survival of 2 years following intensive multimodality treatment (2).

Surgery is only considered in select patients diagnosed with epithelial mesothelioma with a good performance status and optimal cardiopulmonary function (3). The role of exclusive chemoradiotherapy is currently under investigation (4). Presently, radiotherapy is confined to adjuvant settings and is often used in association with chemotherapy, or palliation in advanced stages (5). Therefore, chemotherapy is the primary treatment option for unresectable lesions. First-line treatment with cisplatinpemetrexed is considered the gold standard (6). However, an alternative schedule of cisplatin-raltitrexed may also be administered, which was previously indicated to improve survival in comparison with cisplatin alone during a phase III study (7). A second-line, single agent regimen with gemcitabine or vinorelbine may also be considered in patients with a good performance status $(8,9)$.

Pancreatic cancer is one of most aggressive forms of malignancy, with $<2 \%$ of patients surviving for 5 years or more (10). Surgery has a limited role in the treatment of the disease, with $>80 \%$ of patients diagnosed with unresectable lesions. As with MPM, multimodal chemoradiotherapy is a valid treatment option for cases of pancreatic cancer (11). 

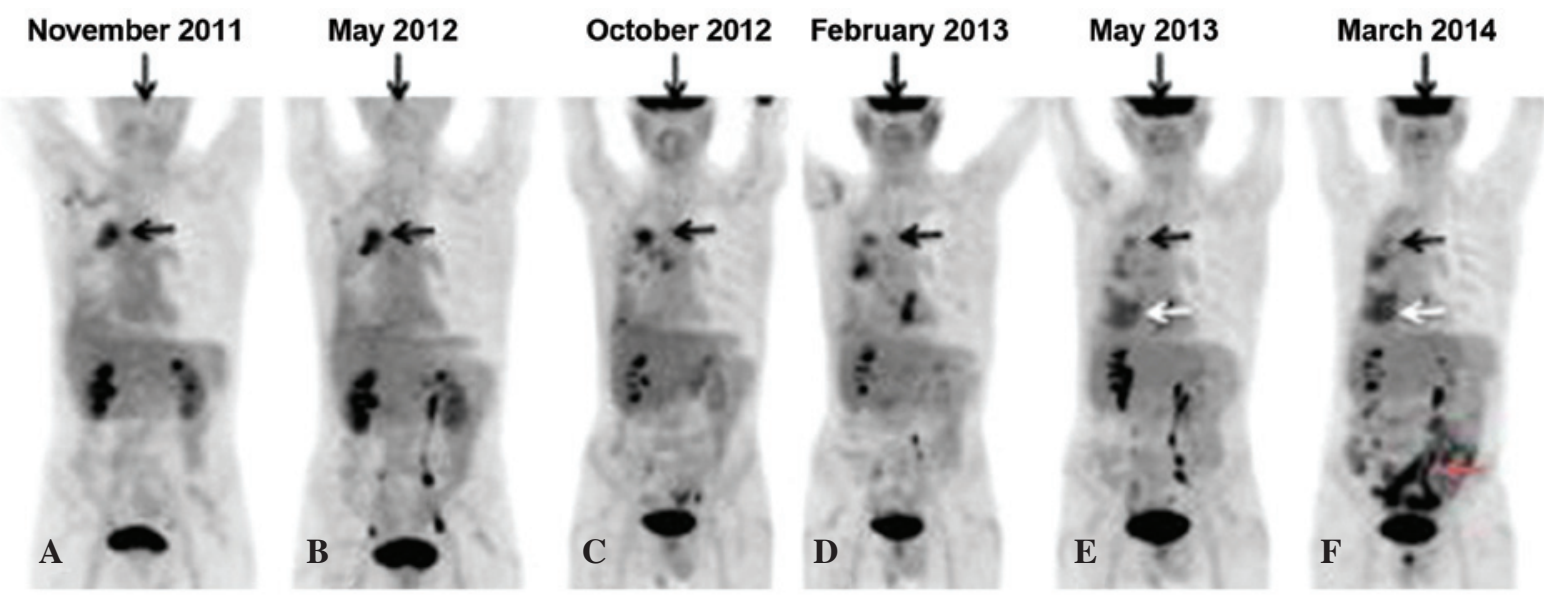

Figure 1. Maximum intensity projection image of PET scans exhibiting 18-fluorodeoxyglucose uptake. Uptake in the right lung decreased following radiotherapy and chemotherapy. Evidence in the last PET scan of the bowel uptake exhibits indirect signs of the upcoming bowel occlusion. Black arrows represent mesothelioma, white arrows represent novel pleural uptake and the red arrow represents bowel uptake. (A) Uptake of the pleural region with no uptake in the pancreas; (B) stability of pleural lesions following 2 chemotherapy cycles; (C) pleural stability following radiotherapy and uptake of pancreatic lesion; (D) light pleural uptake following pancreatic stereo body radiation therapy; (E) pleural uptake increase; and (F) pleural stability and bowel uptake. PET, positron emission tomography.

Over the last two decades, gemcitabine has remained the principal chemotherapeutic drug for the treatment of pancreatic cancer, exhibiting modest clinical benefit with a median patient survival time of 5.4 months (12). A number of chemotherapeutics and targeted agents have been combined with gemcitabine to produce no clinical benefit, including taxane $(13,14)$. Previously, gemcitabine in combination with nab-paclitaxel demonstrated efficient clinical activity in a phase III trial, with statistically significant improvement in median overall survival compared with gemcitabine alone (15). Therefore, the USA Food and Drug Administration approved paclitaxel protein-bound particles (albumin-bound) in combination with gemcitabine for first-line treatment of patients with metastatic adenocarcinoma of the pancreas (16). Alternative therapies include capecitabine, oxaliplatin plus fluorouracil plus leucovorin/capecitabine plus oxaliplatin, and oxaliplatin plus fluorouracil plus leucovorin plus irinotecan (17). Therefore, considering the poor responsiveness to the treatment available to date and the aggressiveness of both tumors, novel therapies are required for the treatment of MPM and pancreatic cancer.

The development of synchronous MPM and other tumors is rare. In a review of 500 patients with asbestos-related MPM, 9 (1.8\%) patients presented with synchronous carcinoma (17). In 6/9 (66.7\%) cases, the secondary tumor was lung carcinoma, including three adenocarcinomas, two squamous cell carcinomas and one small-cell carcinoma, and in $3 / 9$ cases $(33.3 \%)$ the secondary tumor was non-bronchogenic carcinoma, including breast, colonic and pancreatic (18). In a previous study, among 215 cases of malignant pleural mesothelioma, the occurrence of a second malignancy was observed in 32 cases (18.9\%), suggesting that these diseases may share certain etiological factors such as asbestos and others (19).

The onset of bowel metastasis is a rare event in cases of MPM, and to the best of our knowledge, has not been previously described in association with pancreatic cancer.

\section{Case report}

The present study describes the case of a 59-year-old man with a professional history of asbestos exposure, who was diagnosed with locally-advanced MPM in 2011. In November 2011, a computed tomography (CT) scan revealed right pleural effusion and Barety and subcarinal nodes involvement. A suspected nodule, measuring $18 \mathrm{~mm}$ in diameter, at the head of the pancreas was noted. An 18-fluorodeoxyglucose $\left({ }^{18} \mathrm{FDG}\right)$ positron emission tomography (PET) scan was performed (Fig. 1). The scan exhibited uptake of ${ }^{18} \mathrm{FDG}$ in the pleural region [maximum standardized uptake (SUVmax), 2.5] with no uptake in the pancreas (Fig. 1A). Video-assisted thoracoscopy was performed to assist a pleural biopsy, and following analysis, a diagnosis of biphasic mesothelioma was confirmed with immunohistochemical positivity for epithelial specific antigen (EpCAM) and calretinin.

In accordance with the European Organization for Research and Treatment of Cancer prognostic scoring system (20), the patient received 2 cycles of first-line cisplatin-pemetrexed chemotherapy: Pemetrexed, $500 \mathrm{mg} / \mathrm{mq}$ i.v. over $10 \mathrm{~min}$ plus cisplatin, $75 \mathrm{mg} / \mathrm{mq}$ i.v. over $30 \mathrm{~min} 60 \mathrm{~min}$ later, on day 1 every 3 weeks. During February 2012, a tumor assessment reported stability of the pleural disease, an increase in size of the pancreatic lesion $(40 \mathrm{~mm}$ diameter vs. $18 \mathrm{~mm}$ diameter at initial evaluation) and the onset of peripancreatic and axillary node metastasis by $\mathrm{CT}$ scan with contrast $(5-\mathrm{mm}$ thickness slides) (Fig. 2).

Axillary node surgical excision and a laparoscopic pancreatic biopsy were performed, and histological examination of the surgical samples diagnosed an undifferentiated pancreatic adenocarcinoma. The case was evaluated by a multidisciplinary board, and single-agent gemcitabine treatment (gemcitabine, $1,000 \mathrm{mg} / \mathrm{mq}$ iv over $30 \mathrm{~min}$ on days 1 , 8 and 15 of each 28-day cycle) was initiated in May 2012. Following 2 cycles, an ${ }^{18}$ FDG PET scan demonstrated stability of the pleural lesions (SUVmax, 2.6) (Fig. 1B). 


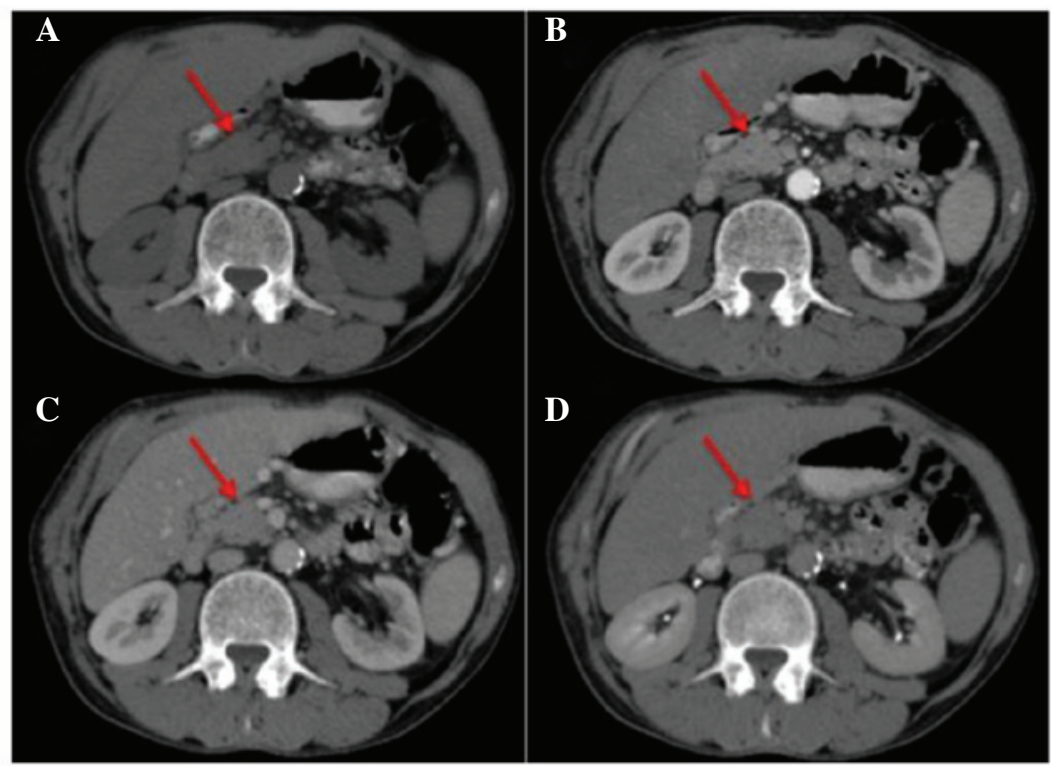

Figure 2. Computed tomography scan of the abdomen showing the pancreatic lesion (red arrow). (A) Without contrast; (B) arterial phase; (C) venous phase; and (D) late phase.

Based on disease stability, the multidisciplinary board advised that multimodal treatment with gemcitabine and concomitant radiotherapy on the pleural lesion should be performed during July 2012. The prescribed dose was $36 \mathrm{~Gy}$, delivered over 12 consecutive fractions at $3 \mathrm{~Gy}$, plus a three-dimensional conformal boost of $3 \mathrm{~Gy}$ to the gross tumor volume delivered using Precise 6 MV Linac system (Elekta AB, Stockholm, Sweden).

Following radiotherapy, the patient continued with single-agent gemcitabine treatment until October 2012, when an ${ }^{18}$ FDG PET scan confirmed pleural stability (SUVmax, 2.5). However, the pancreatic lesion had begun to indicate an uptake of ${ }^{18} \mathrm{FDG}$ (SUVmax, 3.1) (Fig. 1C). Considering the good performance status of the patient, concomitant pancreatic stereotactic body radiation therapy (SBRT) was performed on November 2012. The prescribed dose was $21 \mathrm{~Gy}$ over 3 consecutive fractions using the Precise 15 MV Linac system (Elekta AB). In February 2013, a further ${ }^{18}$ FDG PET scan revealed light pleural uptake (SUVmax, 1.9) (Fig. 1D), and single-agent gemcitabine chemotherapy (gemcitabine, $800 \mathrm{mg} / \mathrm{mq}$ iv over $30 \mathrm{~min}$ on days 1,8 and 15 of each 28-day cycle) was continued.

In May 2013, an ${ }^{18}$ FDG PET scan documented a pleural uptake increase (SUVmax, 3.1) (Fig. 1E). Due to the exacerbation of right hemithorax pain, which was unresponsive to analgesic therapy, the multidisciplinary board proposed that the patient undergo pleural SBRT. The patient received SBRT with a prescribed dose of $25 \mathrm{~Gy}$, over 5 fractions, delivered on 5 alternate days using the Precise 15 MV Linac system. The patient subsequently experienced a significant relief from symptoms, and monochemotherapy with gemcitabine (gemcitabine, $1,000 \mathrm{mg} / \mathrm{mq}$ iv over $30 \mathrm{~min}$ on days 1,8 and 15 of each 28-day cycle) was continued until February 2014.

In March 2014, another ${ }^{18}$ FDG PET scan revealed pleural stability and bowel uptake. The patient was also experiencing abdominal pain and was suspected of having 'acute abdomen'

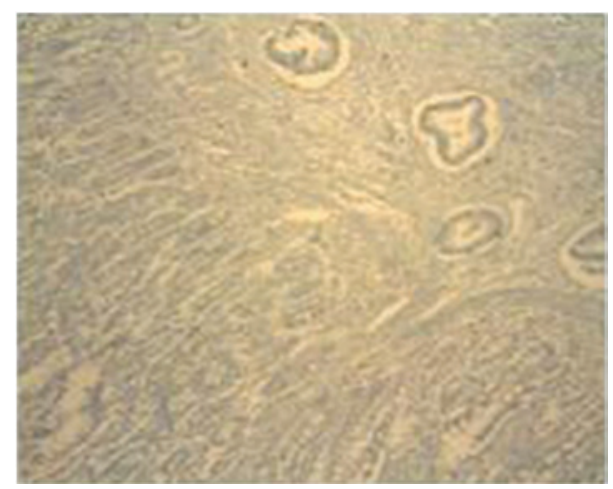

Figure 3. Calretinin immunohistochemistry analysis on the small bowel sample revealed negative staining. Magnification, $\mathrm{x} 10$.

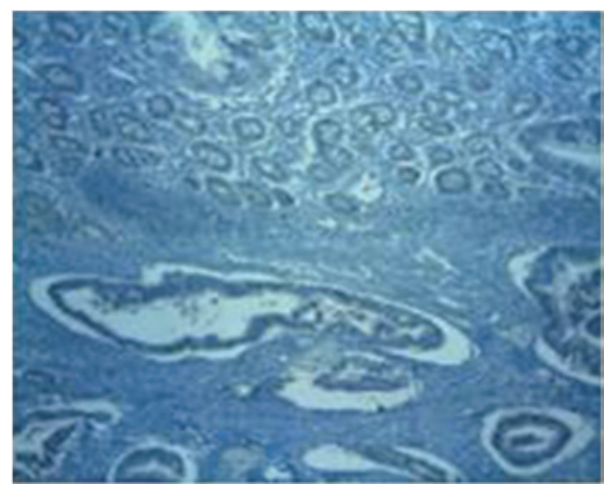

Figure 4. Epithelial specific antigen immunohistochemistry analysis on the small bowel sample revealed negative staining. Magnification, x10.

(Fig. 1F). A CT scan revealed gaseous distension of the small intestine, therefore the patient underwent emergency intestinal resection.

Histological examination was performed at the Department of Pathology of the Second University of Naples 
(Naples, Italy), according to standard local practice (21). Immunohistochemical analysis was performed on 5- $\mu \mathrm{m}$ thick, formalin-fixed, paraffin-embedded tissue sections with the following antibodies: Anti-cytokeratin (CK) 7 (dilution 1:100; catalogue no. ab183344; Abcam, Cambridge, UK), anti-CK20 (dilution 1:100; catalogue no. ab97511; Abcam), anti-caudal type homeobox transcription factor 2 (CDX2; dilution 1:250; catalogue no. ab76541; Abcam), anti-CK5/6 (dilution 1:50; catalogue no. ab17133; Abcam), anti-EpCAM (clone Ber-EP4; dilution 1:250; catalogue no. ab7504; Abcam) and anti-calretinin (dilution 1:50; catalogue no. ab702; Abcam). Analysis of the small intestine sample confirmed the presence of moderately-differentiated adenocarcinoma. Immunohistochemical examination demonstrated that the sample was positive for CK7, negative for CK20 and focally-positive for CDX2. Based on this immunohistochemical profile (negativity for CK20 and positivity for CK7 and CDX2), pathologists excluded an intestinal origin.

Considering the medical history of the patient, a revision of the small intestine sample was performed and immunohistochemistry demonstrated that the tissue was negative for CK5, CK6, CK20, EpCAM and calretinin; therefore, a mesothelial origin of metastasis was also excluded, and the pathologists determined that the pancreatic cancer was an initial form of small bowel metastasis (Figs. 3 and 4).

The multidisciplinary board advised that the patient should begin chemotherapy with gemcitabine and nab-paclitaxel, since previous clinical studies had demonstrated the efficacy of this type of chemotherapy for pancreatic cancer $(15,22)$. The reintroduction of gemcitabine was supported by good results obtained on pleural lesion during previous line of treatment with single agent gemcitabine. The patient continued to receive gemcitabine plus nab-paclitaxel for 3 cycles. Then, for clinical progression, the patient underwent only supportive care for $\sim 2$ months, until succumbing to the disease.

At the time of writing the present case report, the patient was still undergoing treatment. Written informed consent was obtained from the patient for publication of the present study and accompanying images.

\section{Discussion}

MPM is the most common primary tumor of the pleura (1). Exposure to asbestos is the predominant cause of MPM development, with a latency period of at least 35-40 years (2). The incidence of the disease occurs most commonly during the sixth and seventh decades of life with a median survival of 12-18 months regardless of treatment regimen $(1,3)$. Local invasion often affects other contiguous organs, including the pericardium (tamponade and pericardial effusions), the spinal cord (paralysis and back pain) and the contralateral lung (often contralateral pleural effusion) (23). Metastases are usually located at the lymphatic nodes, liver, kidneys and adrenal glands, but are extremely infrequent at the gastrointestinal tract (24). CT and PET scans are important for the diagnosis and assessment of treatment response in cases of mesothelioma.

Regarding pancreatic cancer, no early diagnostic tests or effective treatment options are currently available; therefore, pacreatic cancer remains the fourth leading cause of cancer-associated mortality (25). Typically, pancreatic cancer initially metastasizes to the regional lymph nodes, later to the liver or peritoneal cavity and rarely to the lungs, bone or brain (25).

The present study described the case of patient with an initial diagnosis of MPM and pancreatic carcinoma, with subsequent evidence of a lesion in the small bowel. Based on clinical history, the disease course and immunohistochemical data, it was extremely difficult to identify with certainty whether the bowel lesion was a metastasis from the mesothelioma or pancreatic cancer. Immunohistochemical positivity for CK7 and negativity for CK20 excluded the possibility of an intestinal origin. Therefore, to determine the origin of the bowel metastases, the pathologist performed a revision of the small intestine sample, and further immunohistochemical analysis demonstrated negativity for CK5, CK6, EpCAM and calretinin. This immunohistochemical profile suggested that the pancreatic cancer may have been an initial form of intestinal metastases.

To the best of our knowledge, the current case is the first to describe the development of intestinal metastasis from pancreatic cancer. Certain previous cases of MPM have instead described total involvement of the gastrointestinal lumen by effusion (26-33).

Chen et al (27) reported the case of a 73-year-old man presenting with duodenal metastases from sarcomatoid MPM. Immunohistochemical analysis reported positivity for CK7 and vimentin, and negativity for cluster of differentiation (CD)34, CD117 and calretinin (27).

Liu et al (32) described a patient that presented with MPM with metastases in the jejunum, and the ascending and transverse colon. Immunohistochemical examination on the intestinal sample reported vimentin and cytokeratin positivity and calretinin, CK20 and thyroid transcription factor-1 negativity. This profile was the same as the pleural sample, confirming that the metastases originated from MPM (32).

Based on immunohistochemical results of the present case, a chemotherapy regimen of gemcitabine plus nab-paclitaxel was initiated. The use of gemcitabine was supported by the good results obtained on the pleural lesion during previous lines of treatment with gemcitabine, which was started in May 2012 and exhibited a good control of the disease until March 2014, when it was added to nab-paclitaxel for diagnosis of small bowel metastasis.

The current case highlights the possible role of SBRT in the treatment of oligometastatic diseases, however its application is not yet conventional. If lesions are unable to be surgically resected, radiation therapy may serve a crucial role in disease control on metastatic foci and primary tumors (34). A patient with a good performance status and a lesion $<4 \mathrm{~cm}$ in size is an ideal candidate for SBRT with minimal toxicity on surrounding normal tissue (34). Thus, the role of SBRT in the treatment of metastatic cancer may change from palliative to potentially curative.

The appropriate timing of combining SBRT with chemotherapy remains unclear, considering high risk of recurrence in these patients. It is currently under investigation as to which chemotherapeutic drug should be administered in order to achieve positive tolerability and a radiosensitizing effect (35).

Taking this into account, it is considered that the long-term survival of the current case (33 months) and lasting disease control occurred as a result of a combination of chemotherapy 
regimens, containing active drugs targeting each cancer, and the application of SBRT on the pancreatic cancer lesions.

In conclusion, the present case highlights the requirement of prompt treatment and the use of all available tools to provide an accurate differential diagnosis, even in patients with a presumably poor prognosis. The present case study may implicate future research on the role of SBRT with concomitant chemotherapy in patients with advanced stage disease.

\section{Acknowledgements}

The present study was supported by the Associazione Italiana Per La Ricerca Sul Cancro project (Milan, Italy; grant no. MFAG 2013-N.14392).

\section{References}

1. Teta MJ, Mink PJ, Lau E, Sceurman BK and Foster ED: US mesothelioma patterns 1973-2002: Indicators of change and insights into background rates. Eur J Cancer Prev 17: 525-534, 2008.

2. Krug LM, Pass HI, Rusch VW, Kindler HL, Sugarbaker DJ, Rosenzweig KE, Flores R, Friedberg JS, Pisters K, Monberg M, et al: Multicenter phase II trial of neoadjuvant pemetrexed plus cisplatin followed by extrapleural pneumonectomy and radiation for malignant pleural mesothelioma. J Clin Oncol 27: 3007-3013, 2009.

3. Edwards JG, Abrams KR, Leverment JN, Spyt TJ, Waller DA and O'Byrne KJ: Prognostic factors for malignant mesothelioma in 142 patients: Validation of CALGB and EORTC prognostic scoring systems. Thorax 55: 731-735, 2000.

4. Opitz I: Management of malignant pleural mesothelioma - the European experience. J Thorac Dis 6 (Suppl 2): S238-S252, 2014.

5. Baldini EH: Radiation therapy options for malignant pleural mesothelioma. Semin Thorac Cardiovasc Surg 21: 159-163, 2009

6. Vogelzang NJ, Rusthoven JJ, Symanowski J, Denham C, Kaukel E, Ruffie P, Gatzemeier U, Boyer M, Emri S, Manegold C, et al: Phase III study of pemetrexed in combination with cisplatin versus cisplatin alone in patients with malignant pleural mesothelioma. J Clin Oncol 21: 2636-2644, 2003.

7. van Meerbeeck JP, Gaafar R, Manegold C, Van Klaveren RJ, Van Marck EA, Vincent M, Legrand C, Bottomley A, Debruyne C and Giaccone G; European Organisation for Research and Treatment of Cancer Lung Cancer Group; National Cancer Institute of Canada: Randomized phase III study of cisplatin with or without raltitrexed in patients with malignant pleural mesothelioma: An intergroup study of the European Organisation for Research and Treatment of Cancer Lung Cancer Group and the National Cancer Institute of Canada. J Clin Oncol 23: 6881-6889, 2005.

8. Stebbing J, Powles T, McPherson K, Shamash J, Wells P, Sheaff MT, Slater S, Rudd RM, Fennell D and Steele JP: The efficacy and safety of weekly vinorelbine in relapsed malignant pleural mesothelioma. Lung Cancer 63: 94-97, 2009.

9. van Meerbeeck JP, Baas P, Debruyne C, Groen HJ, Manegold C, Ardizzoni A, Gridelli C, van Marck EA, Lentz M and Giaccone G; European Organization for Research and Treatment of Cancer Lung Cancer Cooperative Group: A phase II study of gemcitabine in patients with malignant pleural mesothelioma. European organization for research and treatment of cancer lung cancer cooperative group. Cancer 85: 2577-2582, 1999.

10. Siegel R, Naishadham D and Jemal A: Cancer statistics, 2013. CA Cancer J Clin 63: 11-30, 2013.

11. Brunner TB and Scott-Brown M: The role of radiotherapy in multimodal treatment of pancreatic carcinoma. Radiat Oncol 5 : 64, 2010.

12. Ghosn M, Kourie HR, El Karak F, Hanna C, Antoun J and Nasr D: Optimum chemotherapy in the management of metastatic pancreatic cancer. World J Gastroenterol 20: 2352-2357, 2014.

13. Moore MJ, Goldstein D, Hamm J, Figer A, Hecht JR, Gallinger S, Au HJ, Murawa P, Walde D, Wolff RA, et al; National Cancer Institute of Canada Clinical Trials Group: Erlotinib plus gemcitabine compared with gemcitabine alone in patients with advanced pancreatic cancer: A phase III trial of the National Cancer Institute of Canada Clinical Trials Group. J Clin Oncol 25: 1960-1966, 2007.
14. Belli $\mathrm{C}$, Cereda $\mathrm{S}$ and Reni M: Role of taxanes in pancreatic cancer. World J Gastroenterol 18: 4457-4465, 2012.

15. Von Hoff DD, Ervin T, Arena FP, Chiorean EG, Infante J, Moore M, Seay T, Tjulandin SA, Ma WW, Saleh MN, et al: Increased survival in pancreatic cancer with nab-paclitaxel plus gemcitabine. N Engl J Med 369: 1691-1703, 2013.

16. Saif MW: U.S. Food and Drug Administration approves paclitaxel protein-bound particles (Abraxane ${ }^{\circledR}$ ) in combination with gemcitabine as first-line treatment of patients with metastatic pancreatic cancer. JOP 14: 686-688, 2013.

17. Kothari N, Saif MW and Kim R: First-line treatment for advanced pancreatic cancer. JOP 14: 129-132, 2013.

18. Attanoos RL, Thomas DH and Gibbs AR: Synchronous diffuse malignant mesothelioma and carcinomas in asbestos-exposed individuals. Histopathology 43: 387-392, 2003.

19. Bianchi C, Bianchi T and Ramani L: Malignant mesothelioma of the pleura and other malignancies in the same patient. Tumori 93: 19-22, 2007.

20. Curran D, Sahmoud T, Therasse P, van Meerbeeck J, Postmus PE and Giaccone G: Prognostic factors in patients with pleural mesothelioma: The European Organization for Research and Treatment of Cancer experience. J Clin Oncol 16: 145-152, 1998.

21. Raucci R, Colonna G, Guerriero E, Capone F, Accardo M, Castello G and Costantini S: Structural and functional studies of the human selenium binding protein-1 and its involvement in hepatocellular carcinoma. Biochim Biophys Acta 1814: 513-522, 2011.

22. Von Hoff DD, Ramanathan RK, Borad MJ, Laheru DA, Smith LS, Wood TE, Korn RL, Desai N, Trieu V, Iglesias JL, et al: Gemcitabine plus nab-paclitaxel is an active regimen in patients with advanced pancreatic cancer: A phase I/II trial. J Clin Oncol 29: 4548-4554, 2011.

23. Robinson BW, Musk AW and Lake RA: Malignant mesothelioma. Lancet 366: 397-408, 2005.

24. Zhang W, Wu X, Wu L, Zhang W and Zhao X: Advances in the diagnosis, treatment and prognosis of malignant pleural mesothelioma. Ann Transl Med 3: 182, 2015.

25. Hidalgo M: Pancreatic cancer. N Engl J Med 362: 1605-1617, 2010.

26. Agaimy A and Wünsch PH: Epithelioid and sarcomatoid malignant pleural mesothelioma in endoscopic gastric biopsies: A diagnostic pitfall. Pathol Res Pract 202: 617-622, 2006.

27. Chen HC, Tsai KB, Wang CS, Hsieh TJ and Hsu JS: Duodenal metastasis of malignant pleural mesothelioma. J Formos Med Assoc 107: 961-964, 2008.

28. Huang YJ, Wu MH and Lin MT: Multiple small-bowel intussusceptions caused by metastatic malignant melanoma. Am J Surg 196: e1-e2, 2008.

29. Eo WK, Kim GY and Choi SI: A case of multiple intussusceptions in the small intestine caused by metastatic renal cell carcinoma. Cancer Res Treat 40: 97-99, 2008.

30. Terashita S, Hirano K, Hirai T, Narabayashi T, Hara Y, Mori H, Endo K and Hirabayashi M: A case of malignant pleural mesothelioma with gastrointestinal metastases which were diagnosed by endoscopic biopsy. Nihon Kokyuki Gakkai Zasshi 47: 133-138, 2009 (In Japanese).

31. Gocho K, Isobe K, Kaburaki K, Honda Y, Mitsuda A, Akasaka Y, Shimada N, Takagi K and Homma S: Malignant pleural mesothelioma presenting as an acute surgical abdomen due to metastatic jejunal perforation. Intern Med 49: 597-601, 2010.

32. Liu H, Cheng YJ, Chen HP, Hwang JC and Chang PC: Multiple bowel intussusceptions from metastatic localized malignant pleural mesothelioma: A case report. World J Gastroenterol 16: 3984-3986, 2010.

33. Martínez Caselles A, Baños Madrid R, Egea Valenzuela J, Molina Martínez J and Carballo Álvarez F: Gastrointestinal bleeding secondary to duodenal metastases of malignant pleural mesothelioma. Rev Esp Enferm Dig 102: 602-603, 2010.

34. de Vin T, Engels B, Gevaert T, Storme G and De Ridder M: Stereotactic radiotherapy for oligometastatic cancer: A prognostic model for survival. Ann Oncol 25: 467-471, 2014.

35. Alongi F, Arcangeli S, Filippi AR, Ricardi U and Scorsetti M: Review and uses of stereotactic body radiation therapy for oligometastases. Oncologist 17: 1100-1107, 2012. 